\title{
Role of kinesiophobia on pain, disability and quality of life in people suffering from chronic musculoskeletal pain: a systematic review
}

\author{
Alejandro Luque-Suarez, ${ }^{1}$ Javier Martinez-Calderon, ${ }^{2}$ Deborah Falla ${ }^{3}$
}

\begin{abstract}
- Additional material is published online only. To view please visit the journal online (http://dx.doi.org/10.1136/ bjsports-2017-098673).
\end{abstract}

1 Departamento de Fisioterapia, Universidad de Malaga, Malaga, Spain

${ }^{2}$ Department of Physiotherapy, University of Malaga, Malaga, Spain

${ }^{3}$ Centre of Precision

Rehabilitation for Spinal Pain (CPR Spine), School of Sport, Exercise and Rehabilitation Sciences, University of Birmingham, Birmingham, UK

Correspondence to Dr Alejandro Luque-Suarez, Departamento de Fisioterapia, Universidad de Malaga, 29071, Spain; aluques@uma.es

Accepted 29 March 2018 Published Online First 17 April 2018
Check for updates

To cite: Luque-Suarez A Martinez-Calderon J, Falla D. Br J Sports Med 2019;53:554-559.

\section{ABSTRACT \\ Objective (1) To explore the level of association} between kinesiophobia and pain, disability and quality of life in people with chronic musculoskeletal pain (CMP) detected via cross-sectional analysis and (2) to analyse the prognostic value of kinesiophobia on pain, disability and quality of life in this population detected via longitudinal analyses.

Design A systematic review of the literature including an appraisal of the risk of bias using the adapted Newcastle Ottawa Scale. A synthesis of the evidence was carried out.

Data sources An electronic search of PubMed, AMED, CINAHL, PsycINFO, PubPsych and grey literature was undertaken from inception to July 2017.

\section{Eligibility criteria for selecting}

studies Observational studies exploring the role of kinesiophobia (measured with the Tampa Scale for Kinesiophobia) on pain, disability and quality of life in people with CMP.

Results Sixty-three articles (mostly cross-sectional) (total sample=10 726) were included. We found strong evidence for an association between a greater degree of kinesiophobia and greater levels of pain intensity and disability and moderate evidence between a greater degree of kinesiophobia and higher levels of pain severity and low quality of life. A greater degree of kinesiophobia predicts the progression of disability overtime, with moderate evidence. A greater degree of kinesiophobia also predicts greater levels of pain severity and low levels of quality of life at 6 months, but with limited evidence. Kinesiophobia does not predict changes in pain intensity.

Summary/conclusions The results of this review encourage clinicians to consider kinesiophobia in their preliminary assessment. More longitudinal studies are needed, as most of the included studies were crosssectional in nature.

Trial registration number CRD42016042641.

\section{INTRODUCTION}

Most people suffer from musculoskeletal pain at least once in their lifetime. ${ }^{1}$ As such, musculoskeletal pain is a highly prevalent and costly condition. ${ }^{1}$ It is the second most common cause of disability in the general population. ${ }^{2}$ There are many established factors (physical, biological, cognitive, behavioural, social, occupational) associated with poor prognosis following the onset of musculoskeletal pain ${ }^{13}$ which helps to explain why many people do not recover after an episode of acute musculoskeletal pain, often resulting in a downward spiral of negative physical, social and psychological consequences. ${ }^{4}$ Among the many biopsychosocial factors which contribute to the experience and impact of pain, negative or maladaptive psychological factors (eg, fear) are among the most important..$^{5-8}$

Fear is considered to be a relevant factor in order to understand how acute pain becomes chronic for some people and why pain and associated outcomes (eg, disability) persist once the tissue damage has healed. ${ }^{9}{ }^{10}$ In this sense, the fear-avoidance (FA) model of pain is one of the frameworks which has received more empirical attention in order to explain the development and persistence of disability following an acute episode of musculoskeletal pain. ${ }^{911}$ According to this model, individuals with a trait tendency to have fear and catastrophic thoughts in response to pain are more at risk of developing chronic musculoskeletal pain (CMP) after an injury compared with individuals who do not have this tendency. ${ }^{9}$ These individuals over-react in response to actual or potential threats, developing avoidance behaviours (eg, hypervigilance) which aim to prevent a new injury/reinjury. ${ }^{9}$ Fear in relation to pain has been described with a variety of conceptual definitions among which pain-related fear, FA beliefs, fear of movement and kinesiophobia are the most commonly used. ${ }^{12}$

Physical inactivity is a potential factor for developing and maintaining $\mathrm{CMP}^{13}$ whereas physical activity has positive benefits in decreasing pain and disability in some CMP conditions, that is, lower limb osteoarthritis. ${ }^{14}$ However, people with CMP often show fear of movement, ${ }^{15}$ which limits the adequate execution of a movement or exercise and leads to more sedentary behaviour. ${ }^{17}$ Such fear imposes a barrier when exercise is prescribed as part of management resulting in significant clinical implications including reduced adherence to treatment and perseverance of a negative experience with pain. ${ }^{11}$

Kinesiophobia (also known as fear of movement) is defined as an excessive, irrational and debilitating fear to carry out a physical movement, due to a feeling of vulnerability to a painful injury or reinjury. ${ }^{18}$ Both constructs are very similar, ${ }^{12}$ and essentially they have the same clinical relevance. While kinesiophobia is usually assessed with the Tampa Scale for Kinesiophobia (TSK), there is not a specific tool to assess fear of movement. ${ }^{12}$ The prevalence of kinesiophobia in persistent pain ranges from $50 \%$ to $70 \% .{ }^{19}{ }^{20}$ It can be acquired through two forms: a direct aversive experience (eg, pain or trauma) or social learning (observation and instruction). ${ }^{21}$ Kinesiophobia may be associated with pain 
and associated outcomes (disability and quality of life) in several ways. First, kinesiophobia alters how people move, possibly with the initial goal to avoid pain. It causes adjustments of motor behaviour which affects the performance of actions related to the management and control of pain and pain-related disability. ${ }^{22}$ Second, the processing of pain and pain-related information in people with CMP could be related to how kinesiophobia is perceived. ${ }^{23}$ Indeed, a greater degree of kinesiophobia predicts greater levels of pain. ${ }^{24}$

The role of kinesiophobia in CMP has been explored extensively. In this regard, a large body of evidence has reported that kinesiophobia is associated with disability, ${ }^{25}$ pain, ${ }^{26-28}$ and quality of life. ${ }^{29}$ Furthermore, longitudinal studies have shown that high levels of kinesiophobia at baseline, predict negative changes in quality of life, ${ }^{30}$ and positive changes in disability, ${ }^{31}$ and pain. ${ }^{3032}$ However, the evidence is inconsistent regarding the strength of the significance and the direction of the findings. ${ }^{3-37}$ A synthesis of the evidence of the association between kinesiophobia and pain, disability and quality of life would contribute to a better understanding and clarification of these relationships in patients with CMP. Moreover, examining the prognostic value of kinesiophobia on the aforementioned outcomes would facilitate a greater understanding of CMP mechanisms and thus permit better clinical decision-making. Clinical decision-making requires ongoing reconciliation of studies that provide different answers to the same question, and clinicians and researchers can also benefit from a summary where uncertainty remains. ${ }^{38}$

A systematic review of the literature will allow stronger conclusions to be reached compared with those achieved by any one study ${ }^{39}$ and will facilitate readers who may have difficulties to capture and review all the evidence provided by primary studies. Hence, the aim of this systematic review was twofold: (1) to explore the level of association between kinesiophobia and pain, disability and quality of life in people with CMP and (2) to analyse the prognostic value of kinesiophobia on pain, disability and quality of life in people with CMP.

\section{METHODS}

This review was conducted in accordance with the Preferred Reporting Items for Systematic Reviews and Meta-Analyses (PRISMA) statement. ${ }^{40}$ The systematic review protocol was registered at the International Prospective Register of Systematic Reviews (PROSPERO: CRD 42016042641). The initial intent of the study, as registered in PROSPERO, was to examine predictive studies only. This approach was abandoned since too few predictive studies existed and we determined that cross-sectional studies and case-control studies were also relevant to include in order to provide additional information on the level of association between kinesiophobia and outcomes related to CMP.

\section{Data sources and search strategy}

The following electronic databases from the inception to February 2017 were searched: PubMed, AMED, CINAHL, PsycINFO and PubPsych. An update of the search strategy was carried out in July 2017 to identify if any new potential studies had been published. A manual search of relevant eligible studies was also carried out. A sensitive search strategy using relevant search terms that were developed from Medical Subject Headings (MeSH), and keywords generated from the subject headings, was used (online supplementary appendix A). The following grey literature databases were explored: JBI COnNECT + , NHS Evidence, New York Academy of Medicine Grey Literature
Report, Explore the British Library, TRIP database, National Guideline Clearinghouse and Open Grey.

\section{Eligibility criteria}

The PECOS (P-patient; E-exposure; C-comparator; O-outcome; S-study design) framework was followed to determine which studies to include in the present systematic review. Each study had to meet the following inclusion criteria:

1. Observational studies (cross-sectional, case-control and longitudinal studies) exploring the predictive role of kinesiophobia in people with CMP and their associations with the outcomes described below. When prospective studies only reported findings from the baseline assessment in sufficient detail, analyses between kinesiophobia and outcome measures were limited to the initial assessment and considered as cross-sectional studies.

2. Studies whose participants were adults diagnosed with CMP, defined in this review as persistent or episodic pain lasting more than 3 months, around the axial skeleton (neck, low back and/or pelvic) or peripheral joints (shoulder, elbow, wrist, knee and/or ankle). In this regard, the ACCTION-APS Pain Taxonomy (AAPT) for chronic pain was used, ${ }^{41}$ including people with diagnoses of chronic myofascial pain, fibromyalgia (FM), chronic widespread pain (eg, chronic fatigue syndrome (CFS)), rheumatoid arthritis, spondyloarthropathies and those with a diagnosis of osteoarthritis. Although the AAPT for chronic pain does not consider spinal pain as part of the musculoskeletal pain group, we decided to include people with chronic axial musculoskeletal pain in the present review.

3. Only studies measuring kinesiophobia with the TSK were included. $^{12}$

4. No restrictions were applied on participants' gender, ethnicity and follow-up duration (in case of longitudinal studies).

5. Studies recruiting participants from the general population, primary, secondary or tertiary care.

6. Only articles written in English were included.

7. Studies were only included if there was at least one association between kinesiophobia and one of the following outcome measures: pain, disability and/or quality of life. With regard to the pain construct, the authors of the present review recognise that the differences between pain intensity and pain severity are narrow. However, in order to facilitate the synthesis of results, we divided both constructs based on a previous literature review ${ }^{42}$ which showed that pain intensity is commonly measured with unidimensional instruments (eg, the numerical rating scale), whereas pain severity is mostly measured with multidimensional instruments (eg, the multidimensional pain inventory), where not only intensity of pain is taken into account.

Exclusion criteria were as follows:

1. Studies of acute pain, subacute pain and chronic non-musculoskeletal pain according to the AAPT.

2. Studies where CMP was associated with a diagnosis of major psychiatric disorders.

3. Studies evaluating kinesiophobia in CMP attributed to previous fracture or exploring this factor before surgery or postsurgery.

4. Studies analysing kinesiophobia in individuals with CMP after trauma.

5. Studies examining the role of kinesiophobia in experimental models of pain. 
6. Studies testing kinesiophobia in the context of a behavioural task or treatment (eg, exposure in vivo).

7. Reviews, clinical studies, case reports, editorial and abstracts.

\section{Study selection}

First, two authors (JMC and ALS) carried out the screening of titles and abstracts. Second, the same authors checked the full text of the included manuscripts. Third, in case of any disagreement, a decision was made by consensus with the participation of a third author (DF). A short checklist was adapted to the present review and was used to guide the selection of relevant studies (see online supplementary appendix B). ${ }^{43}$

\section{Data extraction}

The following relevant data from each study were extracted: study details (first author, year of publication), sample size, characteristics of participants (mean age, pain duration, pain condition), kinesiophobia measures, outcome (pain, disability and quality of life) measures, duration of follow-up (in case of longitudinal studies) and study design. When necessary, an email was sent to the original authors to obtain further information regarding a study's findings.

\section{Quality assessment}

The risk of bias of each included study was evaluated using an adapted version of the Newcastle-Ottawa Scale (NOS). ${ }^{44}$ This adapted version includes four domains of risk of bias assessment: methods for selecting study participants (selection bias), methods to control for confounding (performance bias), statistical methods (detection bias) and methods of exposure and outcome assessment (information bias). Seven items compose the four domains. Each item is scored from 0 (high risk) to 3 (low risk) points. Therefore, the maximum score for each study could be 21 points. Two researchers (JMC and ALS) were involved at this stage. When any disagreement did appear, a third researcher (DF) was consulted and a decision by consensus was carried out. Qualitative analysis of the evidence was carried out involving a rating system (the Modified Cochrane Back and Neck group) with five levels of scientific evidence. ${ }^{45}$ The rating system was based on the methodological quality and the outcome of the studies: (1) strong evidence: consistent findings in two or more low risk of bias studies (score from 14 to 21 on NOS); (2) moderate evidence: consistent findings in one low risk of bias study or two or more moderate risk of bias studies (score from 8 to 13 on NOS); (3) limited evidence: consistent findings in one or more high risk of bias studies (score from 0 to 7 on NOS) or one moderate risk of bias study; (4) lacking evidence: no published studies found; $(v)$ conflicting evidence: inconsistent or contradictory findings within a quality level. Due to the absence of a cut-off in the adapted NOS scale, the authors decided to divide the scores in three sections (mentioned above) according to the levels of evidence included in the Modified Cochrane Back and Neck Group Criteria (low 0-7 on NOS; moderate 8-13 on NOS; strong 14-21 on NOS).

\section{Statistical analysis}

For the primary analysis, studies were grouped per outcomes: disability, pain and quality of life. A meta-analysis could not be carried out due to the presence of heterogeneity in terms of participant's age, sample size, pain condition, outcome measures, version of self-reported kinesiophobia questionnaire (eg, TSK-11 or TSK-17), statistical methods used and study design in most of the studies potentially eligible for conducting a meta-analysis.
Consequently, a descriptive quantitative analysis (the most relevant summary measure with a precision estimate) was provided for each study. For those studies that reported results with several degrees of adjustment for confounders in different models, we extracted the estimate from the model which showed the best adjustment.

\section{RESULTS}

\section{Study characteristics}

A total of 1757 articles were identified through electronic databases, with 148 additional studies identified through reference screening. Six hundred and forty-four titles and abstracts were screened with 345 full-text articles evaluated. The number of studies retrieved from each database and the number of studies excluded in each screening phase are shown in online supplementary figure 1 . The full reference of excluded studies in the last screening $(n=282)$ is reported in online supplementary appendix C. The conflict of interests of included studies are shown in online supplementary appendix D. Of these, 63 observational studies ( 8 longitudinal studies; 50 cross-sectional studies and 5 case-control studies) with a total of 10726 participants (chronic low back pain $(\mathrm{CLBP})=2504 ; \mathrm{FM}=1540$; chronic neck pain $=861$; knee osteoarthritis $=614$; chronic whiplash-associated disorders $=475$; CFS = 385; ankylosing spondylitis $=194$; chronic knee pain $=97$; mixed $=4056$ ) satisfied our inclusion criteria and were included in this review. In case of longitudinal studies, duration of follow-up ranged from 3 to 12 months. Outcomes measures were as follows: disability, $151625-32343746-86$ pain (pain intensity, ${ }^{1525-293134363746-4951-5355-5759606466-69727677798083-8587-91}$ and pain severity ${ }^{153032829293}$ ) and quality of life..$^{28-3047728082899495}$ The characteristics of the included studies are reported in online supplementary appendix E. Eight authors were contacted directly by email to gather any non-published data.

\section{Risk of bias}

The degree to which studies met the risk of bias criteria varied considerably. The risk of bias assessment of all included studies is presented in online supplementary appendix F.

The role of kinesiophobia on pain intensity, pain severity, disability and quality of life in CMP

After analysing the quality of evidence for each outcome included in the present systematic review through the Adapted Cochrane Back and Neck Pain Group Criteria, the evidence was divided between strong and moderate for cross-sectional studies (see table 1) and limited to moderate for longitudinal studies (see table 2). The statistical results for the association between kinesiophobia and pain, disability and quality of life (cross-sectional analysis) are presented in online supplementary appendix $G$ and the predictive value of kinesiophobia on pain, disability and quality of life (longitudinal analysis) is presented in online supplementary appendix $\mathrm{H}$.

\section{The association between kinesiophobia and pain in people with CMP (cross-sectional analysis)}

The association between kinesiophobia and pain intensity based on cross-sectional analyses was evaluated by 38 studies. A total of 21 studies showed a significant association between a greater degree of kinesiophobia and greater levels of pain intensity. $^{15} 294648495153596467-69727683-8587-8991$ There was no significant relationship between kinesiophobia and pain intensity in 13 studies. $^{25343637475255-5760777990}$ One study did not report statistical results about the association between kinesiophobia 
Table 1 Modified Cochrane Back and Neck Pain Group criteria for overall level of evidence based on cross-sectional studies

\begin{tabular}{lccl}
\hline Outcome & $\begin{array}{l}\text { No. } \\
\text { of studies }\end{array}$ & $\begin{array}{l}\text { No. of } \\
\text { participants }\end{array}$ & Level of evidence \\
\hline Kinesiophobia (association with) & & \\
Quality of life & 8 & 1426 & Moderate (negative association) \\
Disability & 46 & 9351 & Strong (positive association) \\
\hline Pain intensity & 38 & 6806 & Strong (positive association) \\
\hline Pain severity & 4 & 767 & Moderate (positive association) \\
\hline
\end{tabular}

Strong evidence: consistent findings in two or more low risk of bias studies (score from 14 to 21 on NOS); moderate evidence: consistent findings in one low risk of bias study or two or more moderate risk of bias studies (score from 8 to 13 on NOS); limited evidence: consistent findings in one or more high risk of bias studies (score from 0 to 7 on NOS) or one moderate risk of bias study; lacking evidence: no published studies found; conflicting evidence: inconsistent or contradictory findings within a quality level.

and pain intensity. ${ }^{80}$ Finally, three studies showed inconsistency in the strength of the significance in their findings. ${ }^{26-28}$ Altug $e t ~ a l^{28}$ showed a non-significant association between kinesiophobia and pain intensity at rest, whereas this association was significant and positive during activity. Lamoth $e t \mathrm{al}^{27}$ showed a non-significant association between kinesiophobia and anticipated pain, whereas this association was significant and positive with regard to actual pain. Lundberg $e \mathrm{al}^{26}$ showed a significant and positive association between kinesiophobia and CLBP, although this association was non-significant when CLBP was diagnosed as non-specific CLBP. Overall the quality of the evidence was strong.

The association between kinesiophobia and pain severity based on cross-sectional analyses was evaluated in four studies. Two studies showed a significant association between a greater degree of kinesiophobia and greater of levels of pain severity. ${ }^{82} 92$ There was no significant relationship between kinesiophobia and pain severity in one study. ${ }^{93}$ One study showed inconsistency in the strength of the significance in their findings. ${ }^{15}$ Bränström et $a l^{15}$ showed a non-significant association between kinesiophobia and pain severity in men, whereas this association was significant and positive in women. Overall the quality of the evidence was moderate.

The predictive value of kinesiophobia on pain in people with CMP (longitudinal analysis)

The predictive value of kinesiophobia on pain intensity in people with CMP was explored in two studies based on longitudinal analyses. ${ }^{316}$ Helminen et $a l^{31}$ reported that the degree of

Table 2 Modified Cochrane Back and Neck Pain Group criteria for overall level of evidence based on longitudinal studies

\begin{tabular}{lccl}
\hline Outcome & $\begin{array}{l}\text { No. of } \\
\text { studies }\end{array}$ & $\begin{array}{l}\text { No. of } \\
\text { participants }\end{array}$ & Level of evidence \\
\hline Kinesiophobia (association with) & \\
Quality of life & 2 & 289 & Limited (negative association) \\
\hline Disability & 7 & 1123 & Moderate (positive association) \\
\hline Pain intensity & 2 & 204 & Conflicting (positive association) \\
\hline Pain severity & 2 & 331 & Limited (positive association) \\
\hline
\end{tabular}

Strong evidence: consistent findings in two or more low risk of bias studies (score from 14 to 21 on NOS); moderate evidence: consistent findings in one low risk of bias study or two or more moderate risk of bias studies (score from 8 to 13 on NOS); limited evidence: consistent findings in one or more high risk of bias studies (score from 0 to 7 on NOS) or one moderate risk of bias study; lacking evidence: no published studies found; conflicting evidence: inconsistent or contradictory findings within a quality level. kinesiophobia at baseline did not predict changes in pain intensity at 12 months after adjusting for age, gender, educational level, comorbidities, body mass index, work status, marital status and disease severity. Koho $e t a l^{70}$ reported that kinesiophobia at baseline did not predict changes in pain intensity at 12 months but no covariates were reported. The overall quality of the evidence was conflicting.

The predictive value of kinesiophobia on pain severity in people with CMP was explored in two studies based on longitudinal analyses. ${ }^{3032}$ Wong et $a^{30}$ reported that baseline kinesiophobia significantly predicted positive changes in pain severity at 6 months, even when adjusted for sociodemographic factors and pain variables (number, duration and intensity). Van den Houte et $a l^{32}$ did not report the predictive value of kinesiophobia on pain severity. The overall quality of the evidence was limited.

\section{The association between kinesiophobia and disability in people with CMP (cross-sectional analysis)}

The association between kinesiophobia and disability was evaluated by 46 studies based on cross-sectional analyses. A total of 30 studies showed a significant association between greater degree of kinesiophobia and greater levels of disability. ${ }^{25} 27-2934$ 46-56 $59646567-6972757678$ 82-84 86 There was no significant relationship between kinesiophobia and disability in 11 studies. ${ }^{3757606263747779-8185}$ One study did not report statistical results on the association between kinesiophobia and disability. ${ }^{58}$ Finally, four studies showed inconsistency in the strength of the significance in their findings. ${ }^{15} 266173$ Bränström et $a l^{15}$ reported a significant and positive association between kinesiophobia and disability in women, whereas this association was non-significant in men. Heuts et al $l^{65}$ found a significant and positive association between kinesiophobia and disability when kinesiophobia was measured with the somatic focus subscale of TSK but not with the activity-avoidance subscale for TSK. Lundberg $e a^{26}$ showed a significant and positive association between kinesiophobia and disability in CLBP, although this association was non-significant when CLBP was diagnosed as non-specific CLBP. De Moraes Vieira et $a l^{46}$ reported a significant and positive association between kinesiophobia and moderate-severe disability, whereas this association was non-significant when the level of disability was minimal. The overall quality of the evidence was strong.

\section{The predictive value of kinesiophobia on disability in people with CMP (longitudinal analysis)}

The predictive value of kinesiophobia on disability in people with CMP was explored in seven studies based on longitudinal analyses. ${ }^{1630-32} 667071$ A greater degree of kinesiophobia at baseline significantly predicted greater levels of disability immediately after the intervention, ${ }^{32}$ and at $3,{ }^{32} 6{ }^{1630}$ and $12^{31}$ months follow-up, even when adjusted for age, ${ }^{30}{ }^{31}$ gender, ${ }^{30} 31$ educational level, ${ }^{31}$ comorbidities, ${ }^{31}$ body mass index,${ }^{31}$ pain variables (number, duration, severity and intensity), ${ }^{1630}$ disability at baseline,${ }^{16}$ work status, ${ }^{31}$ marital status ${ }^{31}$ and disease severity. ${ }^{31}$ One study $^{32}$ reported a significant and positive association between kinesiophobia at baseline and disability after the intervention at 3 months without adjusting for covariates. Furthermore, Lüning Bergsten $e \mathrm{al}^{73}$ found a significant and positive association between changes in kinesiophobia after intervention and changes in disability after intervention without adjusting for any covariates. However, the relationship between changes in kinesiophobia at 6 months and changes in disability at 6 months was not reported. On the other hand, Matos et $a l^{74}$ reported that 
kinesiophobia at baseline did not predict changes in disability at 3 months. No covariates were reported. Koho et al $l^{66}$ also showed that kinesiophobia at baseline did not predict changes in disability at 12 months. No covariates were reported. The overall quality of the evidence was moderate.

\section{The association between kinesiophobia and quality of life in} people with CMP (cross-sectional analysis)

The association between kinesiophobia and quality of life was evaluated in eight studies based on cross-sectional analyses. A total of three studies showed a significant association between a greater levels of kinesiophobia and lower levels of quality of life. ${ }^{297280}$ There was no significant relationship between kinesiophobia and quality of life in one study. ${ }^{96}$ Finally, four studies showed inconsistency in the strength of their findings. ${ }^{28} 478994$ Altug $e{ } a l^{28}$ found a significant and negative association between kinesiophobia and quality of life according to general health, physical function, social function, roles restricted by physical function and bodily pain. However, this association was not significant according to the role restricted by mental function, vitality and mental health. Askary-Ashtiani et $a l^{52}$ showed a significant and negative association between kinesiophobia and quality of life (physical function), although this association was not significant in relation to mental health. Larsson et $a l^{97}$ reported a significant and negative association between kinesiophobia and quality of life when health is poor, whereas this association was not significant when health was very good, good and fair. Nijs and Thielemans ${ }^{94}$ showed a significant and negative association between kinesiophobia and quality of life according to the role limitations due to physical problems, mental health and general health perception. However, this association was not significant according to physical functioning, role limitations due to emotional problems, social functioning, bodily pain and vitality. The overall quality of the evidence was moderate.

\section{The predictive value of kinesiophobia on quality of life in} people with CMP (longitudinal analysis)

The predictive value of kinesiophobia on quality of life in people with CMP was explored in two studies based on longitudinal analyses. ${ }^{3095}$ Wong et $a l^{30}$ reported that greater degree of kinesiophobia at baseline significantly predicted lower levels of quality of life at 6 months, even after being adjusted for sociodemographic factors and pain variables (number, duration and intensity). However, the relationship was only significant with respect to physical function. Orenius et $a l^{95}$ reported that kinesiophobia at baseline did not predict changes in quality of life at 12 months. No covariates were reported. Moreover, the predictive value of changes in kinesiophobia at 12 months and changes in quality of life at 12 months was not reported. The overall quality of the evidence was limited.

\section{DISCUSSION}

The synthesis of the data from the included studies in this systematic review showed the following findings: For the cross-sectional analyses: (1) strong evidence of an association between a greater degree of kinesiophobia and greater levels of pain intensity and disability; (2) moderate evidence for an association between a greater degree of kinesiophobia and greater levels of pain severity; (3) moderate evidence for an association between greater levels of kinesiophobia and lower quality of life. For the longitudinal analyses: (1) a greater degree of kinesiophobia at baseline predicts the progression of disability overtime, with moderate evidence; (2) a greater degree of kinesiophobia at baseline predicts greater levels of pain severity at a 6-month follow-up, with limited evidence; (3) greater levels of kinesiophobia at baseline predict lower quality of life at 6 months, with limited evidence; (4) conflicting evidence was found in support of kinesiophobia as a predictor of changes in pain intensity.

\section{Comparison with other studies}

This is the first synthesis of the evidence evaluating the role of kinesiophobia on pain, disability and quality of life in people with CMP through the analysis of both cross-sectional and longitudinal studies. Fear is a basic emotion, which appears as a reaction to a specific, identifiable and imminent threat (eg, a fall). ${ }^{98}$ It is constituted by three components: interpretation of the stimulus as threatening, increased sympathetic arousal and defensive behaviour. ${ }^{99}$ Escape behaviours are a defensive and adaptive response to stimulus (eg, pain) in the short term (acute phase), ${ }^{100}$ and they can reduce fear levels. However, in the long term (chronic phase), this avoidance activity can become maladaptive. People with CMP are presumed to develop kinesiophobia. ${ }^{15} 16$ They often avoid activities which are assumed to provoke a real or potential injury/reinjury, developing in turn, further physical inactivity. ${ }^{9}$ This fear to carry out certain movements can cause a negative vicious cycle where people with CMP show greater levels of pain, disability and emotional distress, ${ }^{9}$ and as a result, poor quality of life. ${ }^{101}$ Our results support this statement as we identified moderate and strong evidence of associations between a greater degree of kinesiophobia and greater levels of pain, greater levels of disability and poorer quality of life and in addition, moderate evidence that a greater degree of kinesiophobia is a predictor of the progression of disability overtime. Other systematic reviews have evaluated the role of other constructs of fear in acute, subacute and chronic pain. ${ }^{102-105}$ Zale et al ${ }^{102}$ showed how pain-related fear is associated with more severe disability in different acute and chronic pain conditions. Wertli et al explored the prognostic value of FA in low back pain, ${ }^{104}$ and also its role as a mediator of the treatment efficacy in this population. ${ }^{103}$ Their findings showed that FA predicted the delay of recovery in acute and subacute low back pain, favouring the development of chronicity. ${ }^{104}$ Furthermore, FA also predicted poor treatment responses in acute, subacute and early CLBP. ${ }^{103}$ Finally, Kroska ${ }^{105}$ reported how FA is correlated with pain intensity bidirectionally in chronic pain conditions through the analysis of 118 cross-sectional studies.

\section{Strengths and weaknesses of the study}

The present systematic review has several strengths and some limitations that should be mentioned. The strengths are as follows: first, the elaboration and registration of a prespecified protocol on PROSPERO; the use of the PRISMA checklist through the development of this systematic review; the use of the NOS adapted checklist to evaluate the risk of bias of each included study and the Modified Cochrane Back and Neck Pain Group Criteria to analyse the overall quality and strength of the evidence. Second, the inclusion of 63 articles with a total sample of 10726 individuals with CMP exploring the role of kinesiophobia on pain, disability and/ or quality of life allows us to establish a general overview about the role of kinesiophobia in musculoskeletal pain chronicity. As a limitation, even though a long variety of $\mathrm{MeSH}$ terms, grey literature and a manual search were carried out, it is still possible that not all studies were identified. Heterogeneity was present between all included studies (in terms of population, outcome measures, pain conditions, statistical parameters and study design) which limits the opportunity to establish comparisons between studies. Moreover, although mediation analysis should be primarily carried out 
with the aim of identifying causal mechanisms, in order to avoid possible inflation of the results, ${ }^{106}$ none of the included studies specifically evaluated the possible mediating effect of kinesiophobia in CMP and confounding variables were not always explored in all included studies. This review assessed one specific construct of fear (kinesiophobia). Although this construct may share similar characteristics and have similar clinical relevance to other fear constructs (eg, FA beliefs or fear of pain), they are not necessarily interchangeable and readers should consider this when interpreting the results. We selected those studies using the TSK to measure kinesiophobia. Although the TSK has been shown to be the most extended tool to assess this construct, ${ }^{12}$ this may limit the interpretation of our results. We should also recognise several modifications from the initial protocol registered in PROSPERO (CRD 42016042641), as follows: (1) we decided to include cross-sectional and case-control studies, in order to provide information on the prognostic value of kinesiophobia and on the level of association between kinesiophobia and outcomes related to CMP; (2) The Grading of Recommendations Assessment, Development and Evaluation (GRADE) and Risk of bias assessment of prognostic factor studies (QUIPS) were replaced by the adapted version of NOS and by the Modified Cochrane Back and Neck Pain Group Criteria. In our search strategy, we used specific keywords related to pain (eg, chronic pain, low back pain) and did not use 'pain' alone, so we may have missed useful studies. Moreover, we did not include the keyword 'hip pain' in our search strategy, so we may have missed relevant studies here too.

\section{Clinical implications}

Kinesiophobia is known to be a barrier to rehabilitation adherence in different chronic pain conditions. ${ }^{23} 107$ However, it is also considered to be a modifiable factor that may facilitate earlier achievement of pain relief and functional recovery. ${ }^{108}$ In this sense, clinicians should need to identify the presence of kinesiophobia prior to the prescription of any intervention, for example, exercise therapy, since its presence may require a different and more specific approach than standard rehabilitation programmes. Furthermore, although rather speculative, individuals with CMP showing a greater degree of kinesiophobia could be more inclined to search for biomedical explanations and solutions for their pain disorder, due to fear for carrying out exercise or to understand their pain from a biopsychosocial perspective, ${ }^{23}$ giving rise to more comorbid disorders. ${ }^{109}$ In this context, kinesiophobia may cause frustration for both patients and therapists, negatively affecting their therapeutic relationship and limiting rehabilitation efforts. ${ }^{109}$ Therefore, ideally the presence of kinesiophobia should be detected during the first assessment, to plan biopsychosocial treatment strategies focused on the modification of kinesiophobia. This could be achieved through the selection of functional goals, education to manage safe behaviours and graded exposure to feared activities in the form of behavioural experiments (eg, exposure in vivo). ${ }^{9}$

\section{Future research}

In future research: (1) further longitudinal studies are needed analysing prospectively the prognostic value and the mediating role of kinesiophobia in people with CMP; (2) further experimental studies using cognitive-behavioural activities (eg, learnt movements) are warranted in order to manipulate kinesiophobia; (3) experimental studies applying biopsychosocial approaches that methodically address and reduce kinesiophobia are required; (4) studies exploring the role of kinesiophobia on rehabilitation adherence in CMP population are needed; (5) as CMP is a complex multifactorial condition, a number of factors (biological, biomechanical, occupational, contextual, environmental, psychological) besides kinesiophobia may be associated with the development and perpetuation of CMP. These factors should be considered during observational and experimental studies, for example through cluster analysis and mediation analysis, to determine the importance of each factor.

\section{CONCLUSION}

This systematic review revealed that a greater degree of kinesiophobia is associated with greater levels of pain intensity, pain severity and disability as well as lower quality of life, based on the analysis of cross-sectional studies. The analysis of the prognostic role of kinesiophobia showed that greater levels of kinesiophobia at baseline predict higher levels of disability, pain severity and lower quality of life at a 6-month follow-up, while kinesiophobia does not predict changes in pain intensity. The results of this systematic review encourage clinicians to evaluate kinesiophobia in patients with CMP, as the presence of kinesiophobia can impact on adherence to exercise therapy and may require specific management strategies such as the selection of functional goals, education to manage safe behaviours and graded exposure to feared activities. Due to the low number of longitudinal studies found in this review and the low level of the evidence attained in the analysis, causality was not firmly demonstrated; thus, further longitudinal studies are needed.

What is already known

- There is no review on the association between kinesiophobia and pain and disability.

- A systematic review reporting this relationship has not been conducted.

- The prognostic role of kinesiophobia on pain and disability is also unknown.

What are the new findings

A greater degree of kinesiophobia at baseline predicts the progression of disability overtime.

- A greater degree of kinesiophobia is associated with greater pain and disability.

- A greater degree of kinesiophobia is associated with lower quality of life.

Acknowledgements The authors would like to express their gratitude to the University of Malaga for their support through the predoctoral grant obtained by JM-C.

Funding JM-C, PhD student at University of Malaga, is supported by the University of Malaga through a predoctoral grant.

Disclaimer All authors have made a substantial scientific contribution to the study and they are thoroughly familiar with the primary data. All authors have read the complete manuscript and take responsibility for the content and completeness of the manuscript and understand that if the paper, or part of the paper, is found to be faulty or fraudulent, all authors share responsibility.

Competing interests None declared.

Patient consent Not required.

Provenance and peer review Not commissioned; externally peer reviewed.

Data sharing statement Data can be obtained from the corresponding author.

(c) Article author(s) (or their employer(s) unless otherwise stated in the text of the article) 2019. All rights reserved. No commercial use is permitted unless otherwise expressly granted. 


\section{REFERENCES}

1 Cimmino MA, Ferrone C, Cutolo M. Epidemiology of chronic musculoskeletal pain. Best Pract Res Clin Rheumatol 2011:25:173-83.

2 Vos T, Flaxman AD, Naghavi M, et al. Years lived with disability (YLDs) for 1160 sequelae of 289 diseases and injuries 1990-2010: a systematic analysis for the Global Burden of Disease Study 2010. The Lancet 2012;380:2163-96.

3 Artus M, Campbell P, Mallen CD, et al. Generic prognostic factors for musculoskeletal pain in primary care: a systematic review. BMJ Open 2017;7:e012901.

4 Gatchel RJ, Peng YB, Peters ML, et al. The biopsychosocial approach to chronic pain: Scientific advances and future directions. Psychol Bull 2007;133:581-624.

5 Gorczyca R, Filip R, Walczak E. Psychological aspects of pain. Ann Agric Environ Med 2013:1:23-7.

6 Keefe FJ, Rumble ME, Scipio CD, et al. Psychological aspects of persistent pain: current state of the science. J Pain 2004;5:195-211.

7 Severeijns R, Vlaeyen JWS, van den Hout MA, et al. Pain catastrophizing predicts pain intensity, disability, and psychological distress independent of the level of physical impairment. Clin J Pain 2001;17:165-72.

8 Sullivan MJL, Thorn B, Haythornthwaite JA, et al. Theoretical perspectives on the relation between catastrophizing and pain. Clin J Pain 2001;17:52-64.

9 Leeuw M, Goossens MEJB, Linton SJ, et al. The fear-avoidance model of musculoskeletal pain: current state of scientific evidence. J Behav Med 2007;30:77-94.

10 Turk DC, Wilson HD. Fear of pain as a prognostic factor in chronic pain: conceptual models, assessment, and treatment implications. Curr Pain Headache Rep 2010;14:88-95.

11 Vlaeyen JW, Linton SJ. Fear-avoidance and its consequences in chronic musculoskeletal pain: a state of the art. Pain 2000;85:317-32.

12 Lundberg M, Grimby-Ekman A, Verbunt J, et al. Pain-related fear: a critical review of the related measures. Pain Res Treat 2011:2011:1-26.

13 Stubbs B, Patchay S, Soundy A, et al. The avoidance of activities due to fear of falling contributes to sedentary behavior among community-dwelling older adults with chronic musculoskeletal pain: a multisite observational study. Pain Med 2014;15:1861-71.

14 Uthman OA, van der Windt DA, Jordan JL, et al. Exercise for lower limb osteoarthritis: systematic review incorporating trial sequential analysis and network meta-analysis. BMJ 2013;347:f5555.

15 Bränström H, Fahlström M. Kinesiophobia in patients with chronic musculoskeletal pain: Differences between men and women. J Rehabil Med 2008;40:375-80.

16 Picavet HSJ, Vlaeyen JW, Schouten JS. Pain catastrophizing and kinesiophobia: predictors of chronic low back pain. Am J Epidemiol 2002;156:1028-34.

17 Lundberg M, Styf J. Kinesiophobia among physiological overusers with musculoskeletal pain. Eur J Pain 2009;13:655-9.

18 Kori S, Miller R, Todd DD. Kinesiophobia: a new view of chronic pain behavior. Pain Manag 1990;3:35-43.

19 Lundberg M, Larsson M, Östlund $H$, et al. Kinesiophobia among patients with musculoskeletal pain in primary healthcare. J Rehabil Med 2006:38:37-43.

20 Roelofs J, van Breukelen G, Sluiter J, et al. Norming of the tampa scale for kinesiophobia across pain diagnoses and various countries. Pain 2011;152:1090-5.

21 Meier ML, Stämpfli P, Vrana A, et al. Fear avoidance beliefs in back pain-free subjects are reflected by amygdala-cingulate responses. Front Hum Neurosci 2015;9:1-10.

22 Karos K, Meulders A, Gatzounis R, et al. Fear of pain changes movement: Motor behaviour following the acquisition of pain-related fear. European J Pain 2017;21:1432-42.

23 Malfliet A, Van Oosterwijck J, Meeus M, et al. Kinesiophobia and maladaptive coping strategies prevent improvements in pain catastrophizing following pain neuroscience education in fibromyalgia/chronic fatigue syndrome: Pooled results from 2 randomized controlled trials. Physiother Theory Pract 2017;12:1-8.

24 Trost Z, France CR, Thomas JS. Examination of the photograph series of daily activities (PHODA) scale in chronic low back pain patients with high and low kinesiophobia. Pain 2009;141:276-82.

25 Alschuler KN, Hoodin F, Murphy SL, et al. Factors contributing to physical activity in a chronic low back pain clinical sample: A comprehensive analysis using continuous ambulatory monitoring. Pain 2011:152:2521-7.

26 Lundberg M, Frennered K, Hägg 0, et al. The impact of fear-avoidance model variables on disability in patients with specific or nonspecific chronic low back pain. Spine 2011;36:1547-53

27 Lamoth CJC, Meijer OG, Daffertshofer A, et al. Effects of chronic low back pain on trunk coordination and back muscle activity during walking: changes in motor control. Eur Spine J 2006:15:23-40.

28 Altuğ F, Ünal A, Kilavuz G, et al. Investigation of the relationship between kinesiophobia, physical activity level and quality of life in patients with chronic low back pain1. J Back Musculoskelet Rehabil 2016;29:527-31.

29 Oskay D, Tuna Z, Düzgün I, et al. Relationship between kinesiophobia and pain, quality of life, functional status, disease activity, mobility, and depression in patients with ankylosing spondylitis. Turk J Med Sci 2017;47:1340-7.

30 Wong WS, Chow YF, Chen PP, et al. A longitudinal analysis on pain treatment satisfaction among Chinese patients with chronic pain: predictors and association with medical adherence, disability, and quality of life. Quality of Life Research 2015;24:2087-97.

31 Helminen E-E, Sinikallio SH, Valjakka AL, et al. Determinants of pain and functioning in knee osteoarthritis: a one-year prospective study. Clin Rehabil 2016:30:890-900.

32 Van Den Houte M, Luyckx K, Van Oudenhove L, et al. Differentiation progress in a clinical group of fibromyalgia patients during and following a multicomponent treatment program. 2016;98:47-54

33 Antunes RS, de Macedo BG, Amaral TS, et al. Pain, kinesiophobia and quality of life in chronic low back pain and depression. Acta Ortop Bras 2013;21:27-9.

34 Boersma K, Linton SJ. How does persistent pain develop? An analysis of the relationship between psychological variables, pain and function across stages of chronicity. Behav Res Ther 2005:43:1495-507.

35 Dawson AP, Schluter PJ, Hodges PW, et al. Fear of movement, passive coping, manual handling, and severe or radiating pain increase the likelihood of sick leave due to low back pain. Pain 2011;152:1517-24

36 I D, Özgül B, Kuru Colak T, et al. Kinesiophobia in relation to physical activity in chronic neck pain. J Back Musculoskelet Rehabil 2016;29:41-7.

37 Dimitriadis Z, Kapreli E, Strimpakos N, et al. Do psychological states associate with pain and disability in chronic neck pain patients? J Back Musculoskelet Rehabil 2015;28:797-802

38 Garg AX, Hackam D, Tonelli M. Systematic review and meta-analysis: when one study is just not enough. Clin J Am Soc Nephrol 2008;3:253-60.

39 Chan ME, Arvey RD. Meta-Analysis and the Development of Knowledge. Perspect Psychol Sci 2012;7:79-92.

40 Liberati A, Altman DG, Tetzlaff J, et al. The PRISMA statement for reporting systematic reviews and meta-analyses of studies that evaluate health care interventions: explanation and elaboration. J Clin Epidemiol 2009:62:e1-e34.

41 Dworkin RH, Bruehl S, Fillingim RB, et al. Multidimensional Diagnostic Criteria for Chronic Pain: Introduction to the ACTTION-American Pain Society Pain Taxonomy (AAPT). J Pain 2016;17:T1-T9.

42 Younger J, McCue R, Mackey S. Pain outcomes: a brief review of instruments and techniques. Curr Pain Headache Rep 2009;13:39-43.

43 Adom T, Puoane T, De Villiers A, et al. Prevalence of obesity and overweight in African learners: a protocol for systematic review and meta-analysis. BMJ Open 2017;7:e013538.

44 Bawor M, Dennis BB, Bhalerao A, et al. Sex differences in outcomes of methadone maintenance treatment for opioid use disorder: a systematic reviewand metaanalysis. CMAJ Open 2015:3:E344-E351.

45 Konieczka C, Gibson C, Russett L, et al. What is the reliability of clinical measurement tests for humeral head position? A systematic review. J Hand Ther 2017:30:420-31.

46 de Moraes Vieira Érica Brandão, de Góes Salvetti M, Damiani LP, et al. Self-efficacy and fear avoidance beliefs in chronic low back pain patients: coexistence and associated factors. Pain Manag Nurs 2014;15:593-602.

47 Turk DC, Robinson JP, Burwinkle T. Prevalence of fear of pain and activity in patients with fibromyalgia syndrome. J Pain 2004;5:483-90.

48 Carvalho FA, Maher CG, Franco MR, et al. Fear of movement is not associated with objective and subjective physical activity levels in chronic nonspecific low back pain. Arch Phys Med Rehabil 2017:98:96-104.

49 Meeus M, Nijs J, Van Mol E, et al. Role of psychological aspects in both chronic pain and in daily functioning in chronic fatigue syndrome: a prospective longitudinal study. Clin Rheumatol 2012;31:921-9.

50 Lambin DI, Thibault P, Simmonds M, et al. Repetition-induced activity-related summation of pain in patients with fibromyalgia. Pain 2011;152:1424-30.

51 Areeudomwong P, Buttagat V. Reliability and validity of the cross-culturally adapted thai version of the tampa scale for kinesiophobia in knee osteoarthritis patients. Malays J Med Sci 2017;24:61-7.

52 Askary-Ashtiani A, Ebrahimi-Takamejani I, Torkaman G, et al. Reliability and validity of the persian versions of the fear avoidance beliefs questionnaire and tampa scale of kinesiophobia in patients with neck pain. Spine 2014;39:E109 5-E1102.

53 French DJ, France CR, Vigneau F, et al. Fear of movement/(re)injury in chronic pain: a psychometric assessment of the original English version of the Tampa scale for kinesiophobia (TSK). Pain 2007:127:42-51.

54 George SZ, Calley D, Valencia C, et al. Clinical investigation of pain-related fear and pain catastrophizing for patients with low back pain. Clin J Pain 2011:27:108-15.

55 Roelofs J, Goubert L, Peters ML, et al. The Tampa Scale for Kinesiophobia: further examination of psychometric properties in patients with chronic low back pain and fibromyalgia. European Journal of Pain 2004;8:495-502.

56 Roelofs J, Sluiter JK, Frings-Dresen MHW, et al. Fear of movement and (re)injury in chronic musculoskeletal pain: Evidence for an invariant two-factor model of the Tampa Scale for Kinesiophobia across pain diagnoses and Dutch, Swedish, and Canadian samples. Pain 2007;131:181-90.

57 Rusu AC, Kreddig N, Hallner D, et al. Fear of movement/(Re)injury in low back pain: confirmatory validation of a German version of the Tampa Scale for Kinesiophobia. BMC Musculoskelet Disord 2014;15:1-9.

58 Woby SR, Roach NK, Urmston M, et al. Psychometric properties of the TSK-11: a shortened version of the Tampa Scale for Kinesiophobia. Pain 2005;117:137-44. 
$59 \mathrm{Er} \mathrm{G}$, Angln E. Determining the relationship of kinesiophobia with respiratory functions and functional capacity in ankylosing spondylitis. Medicine 2017;96:e7486.

60 Akbari F, Dehghani M, Khatibi A, et al. Incorporating family function into chronic pain disability: the role of catastrophizing. Pain Res Manag 2016;2016:1-9.

61 Domenech J, Sanchis-Alfonso V, López L, et al. Influence of kinesiophobia and catastrophizing on pain and disability in anterior knee pain patients. Knee Surgery, Sport Traumatol Arthrosc 2013;21:1562-8

62 Elfving B, Andersson T, Grooten WJ. Low levels of physical activity in back pain patients are associated with high levels of fear-avoidance beliefs and pain catastrophizing. Physiother Res Int 2007;12:14-24.

63 Ferrari S, Chiarotto A, Pellizzer M, et al. Pain self-efficacy and fear of movement are similarly associated with pain intensity and disability in italian patients with chronic low back pain. Pain Pract 2016;16:1040-7.

64 Franklin ZC, Smith NC, Fowler NE. Influence of defensiveness on disability in a chronic musculoskeletal pain population. Pain Practice 2016;16:882-9.

65 Heuts PHTG, Vlaeyen JWS, Roelofs J, et al. Pain-related fear and daily functioning in patients with osteoarthritis. Pain 2004;110:228-35.

66 Howell ER, Hudes K, Vernon H, et al. Relationships between cervical range of motion, self-rated disability and fear of movement beliefs in chronic neck pain patients. J Musculoskelet Pain 2012;20:18-24.

67 Huijnen IPJ, Verbunt JA, Roelofs J, et al. The disabling role of fluctuations in physical activity in patients with chronic low back pain. Eur J Pain 2009;13:1076-9.

68 Huis 't Veld RM, Vollenbroek-Hutten MM, Groothuis-Oudshoorn KC, et al. The role of the fear-avoidance model in female workers with neck-shoulder pain related to computer work. Clin J Pain 2007;23:28-34.

69 Johansen JB, Røe C, Bakke ES, et al. The determinants of function and disability in neck patients referred to a specialized outpatient clinic. Clin J Pain 2013;29:1029-35.

70 Koho P, Orenius T, Kautiainen $\mathrm{H}$, et al. Association of fear of movement and leisure-time physical activity among patients with chronic pain. J Rehabil Med 2011:43:794-9.

71 Lewis $\mathrm{S}$, Holmes P, Woby $\mathrm{S}$, et al. The relationships between measures of stature recovery, muscle activity and psychological factors in patients with chronic low back pain. Man Ther 2012;17:27-33.

72 Lopez-De-Uralde-Villanueva I, Beltran-Alacreu H, Fernández-Carnero J, et al. Differences in neural mechanosensitivity between patients with chronic nonspecific neck pain with and without neuropathic features. A descriptive cross-sectional studzy. Pain Med 2016;17:136-48.

73 Lüning Bergsten C, Lundberg M, Lindberg P, et al. Change in kinesiophobia and its relation to activity limitation after multidisciplinary rehabilitation in patients with chronic back pain. Disabil Rehabil 2012;34:852-8.

74 Matos M, Bernardes SF, Goubert L, et al. Buffer or amplifier? Longitudinal effects of social support for functional autonomy/dependence on older adults' chronic pain experiences. Health Psychol 2017;36:1195-206.

75 Nijs J, Vanherberghen K, Duquet W, et al. Chronic fatigue syndrome: lack of association between pain-related fear of movement and exercise capacity and disability. Phys Ther 2004;84:696-705.

76 Nijs J, De Meirleir K, Duquet W. Kinesiophobia in chronic fatigue syndrome: assessment and associations with disability. Arch Phys Med Rehabil 2004;85:1586-92.

77 Russek L, Gardner S, Maguire K, et al. A cross-sectional survey assessing sources of movement-related fear among people with fibromyalgia syndrome. Clin Rheumatol 2015;34:1109-19.

78 Saavedra-Hernández M, Castro-Sánchez AM, Cuesta-Vargas Al, et al. The contribution of previous episodes of pain, pain intensity, physical impairment, and pain-related fear to disability in patients with chronic mechanical neck pain. Am J Phys Med Rehabil 2012;91:1070-6.

79 Schiphorst Preuper HR, Reneman MF, Boonstra AM, et al. Relationship between psychological factors and performance-based and self-reported disability in chronic low back pain. Eur Spine J 2008;17:1448-56.

80 Sinikallio SH, Helminen EE, Valjakka AL, et al. Multiple psychological factors are associated with poorer functioning in a sample of community-dwelling knee osteoarthritis patients. J Clin Rheumatol 2014;20:261-7.

81 Thomas E-N, Pers Y-M, Mercier G, et al. The importance of fear, beliefs, catastrophizing and kinesiophobia in chronic low back pain rehabilitation. Ann Phys Rehabil Med 2010;53:3-14.

82 Thompson DP, Moula K, Woby SR. Are fear of movement, self-efficacy beliefs and fear of falling associated with levels of disability in people with osteoarthritis of the knee? A cross sectional study. Musculoskeletal Care 2017;15.
83 Vernon $\mathrm{H}$, Guerriero R, Kavanaugh S, et al. Psychological factors in the use of the neck disability index in chronic whiplash patients. Spine 2010;35:E16-E21.

84 Vernon H, Guerriero R, Soave D, et al. The relationship between self-rated disability, fear-avoidance beliefs, and nonorganic signs in patients with chronic whiplashassociated disorder. J Manipulative Physiol Ther 2011;34:506-13.

85 Vernon H, Guerriero R, Kavanaugh S, et al. Self-rated disability, fear-avoidance beliefs, nonorganic pain behaviors are important mediators of ranges of active motion in chronic whiplash patients. Disabil Rehabil 2013;35:1954-60.

86 Vincent HK, Omli MR, Day T, et al. Fear of movement, quality of life, and self-reported disability in obese patients with chronic lumbar pain. Pain Med 2011;12:154-64.

87 Vlaeyen JWS, Kole-Snijders AMJ, Rotteveel AM, et al. The role of fear of movement/ (re)injury in pain disability. J Occup Rehabil 1995;5:235-52.

88 Damsgard E, Thrane G, Anke A, et al. Activity-related pain in patients with chronic musculoskeletal disorders. Disabil Rehabil 2010;32:1428-37.

89 Larsson C, Ekvall Hansson E, Sundquist K, et al. Kinesiophobia and its relation to pain characteristics and cognitive affective variables in older adults with chronic pain. BMC Geriatr 2016;16:128.

90 Vlaeyen JWS, Kole-Snijders AMJ, Boeren RGB, et al. Fear of movement/(re) injury in chronic low back pain and its relation to behavioral performance. Pain 1995:62:363-72

91 Falla D, Peolsson A, Peterson G, et al. Perceived pain extent is associated with disability, depression and self-efficacy in individuals with whiplash-associated disorders. Eur J Pain 2016:20:1490-501.

92 Sullivan MJL, Thibault P, Andrikonyte J, et al. Psychological influences on repetitioninduced summation of activity-related pain in patients with chronic low back pain. Pain 2009;141:70-8.

93 Thibault P, Loisel P, Durand M-J, et al. Psychological predictors of pain expression and activity intolerance in chronic pain patients. Pain 2008;139:47-54.

94 Nijs J, Thielemans A. Kinesiophobia and symptomatology in chronic fatigue syndrome: a psychometric study of two questionnaires. Psychol Psychother 2008:81:273-83.

95 Orenius T, Koskela T, Koho P, et al. Anxiety and depression are independent predictors of quality of life of patients with chronic musculoskeletal pain. J Health Psychol 2013;18:167-75

96 Gatchel RJ, Peng YB, Peters ML, et al. The biopsychosocial approach to chronic pain: scientific advances and future directions. Psychol Bull 2007;133:581-624.

97 Larsson C, Ekvall Hansson E, Sundquist K, et al. Impact of pain characteristics and fear-avoidance beliefs on physical activity levels among older adults with chronic pain: a population-based, longitudinal study. BMC Geriatr 2016;16:50.

98 Rachman S. Anxiety. Hove, East Sussex, UK: Psychology Press, 1998

99 Rachman S, Hodgson R. I. Synchrony and desynchrony in fear and avoidance. Behav Res Ther 1974:12:311-8.

100 Steimer T. The biology of fear- and anxiety-related behaviors. Dialogues Clin Neurosci 2002:4:231-49.

101 Shim E, Hahm B, Dj G, et al. Modeling quality of life in patients with rheumatic diseases : the role of pain catastrophizing, fear-avoidance beliefs, physical disability, and depression. Disabil Rehabil 2017;14:1-8.

102 Zale EL, Lange KL, Fields SA, et al. The relation between pain-related fear and disability: a meta-analysis. J Pain 2013;14:1019-30.

103 Wertli MM, Rasmussen-Barr E, Held U, et al. Fear-avoidance beliefs-a moderator of treatment efficacy in patients with low back pain: a systematic review. Spine $J$ 2014; 14:2658-78.

104 Wertli MM, Rasmussen-Barr E, Weiser S, et al. The role of fear avoidance beliefs as a prognostic factor for outcome in patients with nonspecific low back pain: a systematic review. Spine J 2014;14:816-36.

105 Kroska EB. A meta-analysis of fear-avoidance and pain intensity: The paradox of chronic pain. Scand J Pain 2016:13:43-58.

106 Imai K, Keele L, Tingley D. A general approach to causal mediation analysis. Psychol Methods 2010:15:309-34.

107 Bäck M, Cider $\AA$, Herlitz J, et al. Kinesiophobia mediates the influences on attendance at exercise-based cardiac rehabilitation in patients with coronary artery disease. Physiother Theory Pract 2016;32:571-80.

108 George SZ, Lentz TA, Zeppieri G, et al. Analysis of shortened versions of the tampa scale for kinesiophobia and pain catastrophizing scale for patients after anterior cruciate ligament reconstruction. Clin J Pain 2012;28:73-80.

109 Hadjistavropoulos HD, Kowalyk KM. Patient therapist relationships among patients with pain-related fear. In: Asmundson G, Vlaeyen J, Crombez G, eds. Understanding and treating fear of pain. New York: Oxford University Press, 2004. 